\title{
A Chebyshev Polynomial-Based Conditional Privacy- Preserving Authentication and Group-Key Agreement Scheme for VANET
}

Jiyun Yang ( $\nabla$ yangjy@cqu.edu.cn )

Chongqing University

Jiamin Deng

Chongqing University

Tao Xiang

Chongqing University

Bo Tang

Chongqing University

\section{Research Article}

Keywords: Chebyshev polynomial, Conditional privacy, Chinese remainder theorem, VANET

Posted Date: June 14th, 2021

DOI: https://doi.org/10.21203/rs.3.rs-550221/v1

License: (a) (i) This work is licensed under a Creative Commons Attribution 4.0 International License.

Read Full License

Version of Record: A version of this preprint was published at Nonlinear Dynamics on October 25th, 2021. See the published version at https://doi.org/10.1007/s11071-021-06898-y. 


\title{
A Chebyshev polynomial-based conditional privacy-preserving authentication and group-key agreement scheme for VANET
}

\author{
Jiyun Yang - Jiamin Deng · Tao Xiang · Bo Tang
}

Received: date / Accepted: date

\begin{abstract}
Vehicle ad hoc network (VANET) is an open communication environment. Any user can broadcast messages, which means that it can be easily attacked by malicious users. Therefore, the authentication of vehicles is needed. In this paper, we propose a Chebyshev polynomial-based conditional privacypreserving authentication and group-key agreement scheme for VANET. Specifically, we solve three problems in VANET: (1) we improve the effectiveness of TA by using Chebyshev polynomial to authenticate vehicles; (2) we reduce the computational burden of TA by using Chinese remainder theorem to manage group members; (3) we provide conditional privacy for users by using traceable pseudonym scheme. Theoretical and experimental results show that the proposed scheme is more efficient than existing related work.
\end{abstract}

Keywords Chebyshev polynomial · Conditional privacy · Chinese remainder theorem · VANET

Jiyun Yang

174, Shazheng street, Shapingba district

Tel.: +8613983793873

E-mail: yangjy@cqu.edu.cn

Jiamin Deng

174, Shazheng street, Shapingba district

Tao Xiang

174, Shazheng street, Shapingba district

Bo Tang

174, Shazheng street, Shapingba district

\section{Introduction}

With the development of automobile industry and the improvement of economic level, the number of cars in the city continues to grow, and traffic jams and traffic accidents occur frequently. The modern intelligent transportation system (ITS) is a most promising direction to provide an efficient way to manage the cars in the city[11]. As a cornerstone of ITS, vehicular ad-hoc network (VANET), which is a special mobile self-organizing network, allows vehicles to communicate with each other via vehicle-to-vehicle (V2V) communications and communicate with roadside units (RSUs) via vehicle-to-infrastructure (V2I) communications. Both V2V and V2I communications are based on a dedicated short range communication (DSRC) protocol[5][3].

Among many in-vehicle applications, safety applications (such as coordinated driving, collision avoidance, lane change warning, congestion avoidance) are one of the most concerned and important applications in VANET. To implement this application, real-time traffic-related information needs to be collected and processed in a timely manner. According to the DSRC protocol, the vehicle broadcasts its own traffic status information, such as speed, direction, and road conditions every 100 to $300 \mathrm{~ms}$ during driving[8]. Using this type of information, vehicles, RSUs, and traffic control application centers can achieve collision avoidance and road optimization, thereby improving road safety and traffic efficiency.

However, in this form of wireless communication, VANET may receive various attacks such as malicious detection, interception, modification, and replay. Message authentication is an effective defense against malicious attacks, yet many types of existing authentica- 
tion schemes still have various problems in terms of efficiency and security.

In this paper, we propose a conditional privacypreserving authentication scheme based on Chebyshev chaotic mapping. Our method has following two advantages. First, it satisfies various security requirements including conditional privacy, and can resist modification attack, replay attack and so on. Second, it reduces the computational overhead compared with methods based on elliptic cryptosystems or bilinear pairing and, as a result, it improves the effectiveness of authentication.

In addition, we propose a group key distribution method. Vehicles can communicate freely after authentication. Our proposed method is based on the Chinese residual theorem, which can effectively manage vehicles' entering and leaving the group. It also ensures forward and backward security.

The main contributions of this paper are summarized as follows.

1) A novel conditional privacy-preserving authentication scheme is proposed, which is implemented using Chebyshev polynomial instead of bilinear pairing or elliptic cryptosystems with high computational cost.

2) An efficient group key distribution method based on the Chinese residual theorem is proposed for legitimate vehicles to join and leave a group, which achieves V2V communications while providing location privacy.

3) We provide a formal proof based on BAN Logic to prove the security of our scheme and conduct comprehensive analysis of the performance of our scheme in terms of computation and communication costs.

The remainder of this paper is organized as follows. Section II summarizes the previous works in the literature. The system model, security requirement and Chebyshev polynomial are presented in Section III. We describe our proposed scheme in Section IV. Section V analyzes the security strength of our proposed scheme. Section VI provides the performance evaluation metrics and results of our proposed algorithm with the other existing key management schemes. Section VIII gives concluding remarks.

\section{Related Works}

In order to provide security, efficiency, and conditional privacy for VANET, many available techniques about authentication in VANET are designed. We briefly introduce some representative related works from the following two aspects.

Authentication mechanism .

In order to solve the security and privacy issues in
VANET, authentication mechanism has been widely researched. In 2007, Raya and Hubaux [10] designed a conditional privacy-preserving model using anonymous certificates to implement authentication and integrity functions by public key infrastructure (PKI). In the scheme, message receiver cannot track the owner of the keys. However, a large storage space is needed. OBU needs to be equipped with a large number of public and private key pairs and corresponding anonymous certificates. Moreover, it requires a large number of CRL checks, which leads to DoS attack because of computation and communication overhead. In 2008, Zhang et al. [16] proposed an identity-based PKI conditional privacy authentication scheme, in which vehicles and RSUs do not need to store any certificates. However, the scheme is vulnerable to replay attacks and could not satisfy non-repudiation. In 2015, He et al. [4] proposed a CPA scheme that does not rely on bilinear pairing, greatly reducing computational overhead. In 2016, Lo et al. [9] proposed an identities-based CPA scheme that uses elliptic curves to meet privacy requirements, however, the cost of computation is still unsatisfactory. In 2017, Vijayakumar et al. [1] proposed an efficient anonymous authentication scheme by using bilinear pairing to achieve conditional privacy. However, the efficiency of this scheme is relatively low.

Our scheme adopts Chebyshev for authentication instead of time-consuming bilinear pairing operation and mapping to point operation, which improves the effectiveness of TA authentication and satisfies various security requirements including non-repudiation, forward and backward security and can resist modification attack and so on.

\section{Group key agreement .}

Group key agreement provides secure channel for V2V communication in VANET. In 2015, Vijayakumar et al. [13] proposed a dual authentication and key management protocol that uses the Chinese residual theorem to manage the entry and departure of vehicles within the scope of the RSU. The scheme only needs to update a small amount of information and has high computational efficiency. However, it does not implement non-repudiation. Using a pseudonym or digital signature can provide a way to implement nonrepudiation authentication. In 2019, Li et al. [6] proposed an anonymous conditional privacy protection authentication scheme for VANET based on message authentication code (MAC). With verifiable secret sharing (VSS), a vehicle can obtain a group key for message generation and authentication. However, it cannot implement the key update operation, and it always uses the same group key. Therefore, the forward and backward 
security is not guaranteed. The main limitation of these existing efforts is the performance degradation caused by the computational complexity involved in rekey operations. In the key update strategy, the join and leave protocol is an issue. The main limitation of key updating is the high computational complexity.

The CRT-based authentication scheme proposed in this paper is more efficient compared with most existing authentication and group key management schemes. The computation complexity of the TA and vehicles is reduced substantially by minimizing the number of arithmetic operations taken by the TA and vehicles. The number of key values stored by VANET users is also minimized in this work compared with existing group key management algorithms.

\section{Preliminary}

In this section, we briefly introduce some preliminaries that will be used in this paper. Chebyshev chaotic maps based cryptography can be used to provide an efficient and secure way for authentication.

\subsection{Chebyshev Polynomial}

Chebyshev Polynomial and Its Properties. For integer $n$ and a variable $x \in[-1,+1]$, Chebyshev polynomial $T_{n}(x):[-1,+1] \rightarrow[-1,+1]$, degree $n$, is defined as

$T_{n}(x)= \begin{cases}\cos (n \cdot \arccos (x)) & \text { if } x \in[-1,1] \\ \cos (n \theta) & \text { if } x=\cos \theta, \theta \in[0, \pi] .\end{cases}$

The recursive formulation is

$T_{n}(x)= \begin{cases}1 & \text { if } n=0 \\ x & \text { if } n=1 \\ 2 x T_{n-1}(x)-T_{n-2}(x) & \text { if } n \geq 2 .\end{cases}$

Definition 1. One of most important property from cryptographic perspective is that the Chebyshev polynomial exhibits the semi-group property[17], as follows: $T_{n}(x) \equiv\left(2 x T_{n-1}(x)-T_{n-2}(x)\right)(\bmod p)$, where $n \geq 2, x \in(-\infty,+\infty), p$ is a prime number. Obviously, $T_{r}\left(T_{s}(x)\right) \equiv T_{s r}(x) \equiv T_{s}\left(T_{r}(x)\right)(\bmod p)$, where $\mathrm{r}$ and $\mathrm{s}$ are two positive integers, $s, r \in Z_{p}^{*}$.

Definition 2. Chaotic Maps based Computational Diffie Hellman Problem (CMDHP). Given the values of $x \in(-\infty,+\infty), T_{r}(x) \bmod p, T_{s}(x) \bmod p$ and a large prime $p$, it is intractable to compute $T_{r s}(x) \bmod p$.

\subsection{Hash Function based on Chaotic Map}

Chaotic maps can be used in constructing hash function because of their properties, such as parametersensitivity and random-similarity. The process of algorithm is as following [15]:

INPUT: bit string y of arbitrary length

OUTPUT: 128-bit hash value

The advantage of Chaos hash is that it can reduce basic operations. We use Chebyshev-based sequences to construct Hash to reduce the computational storage complexity of OBU, thereby improving the operation effectiveness.

\subsection{Chinese Remainder Theorem}

The Chinese remainder theorem states that if one knows the remainders of the Euclidean division of an integer $\mathrm{n}$ by several integers, then one can determine uniquely the remainder of the division of $n$ by the product of these integers, under the condition that the divisors are pairwise coprime[18].

Let $k_{1}, k_{2}, k_{3}, \ldots, k_{n}$ be pairwise relatively prime positive integers, and let $a_{1}, a_{2}, a_{3}, \ldots$, an be positive integers. Then, CRT states that the pair of congruences

$$
\begin{aligned}
& X \equiv a_{1} \bmod k_{1} \\
& X \equiv a_{2} \bmod k_{2} \\
& \cdots \\
& X \equiv a_{n} \bmod k_{n}
\end{aligned}
$$

has a unique solution mod $\partial_{g}=\prod_{i=1}^{n}\left(k_{i}\right)$

To compute the unique solution, the TA can compute the value as shown in equation.

$X=\left(\sum_{i=1}^{n} \alpha_{i} \beta_{i} \gamma_{i}\right) \bmod \partial_{g}$

Where, $\beta_{i}=\frac{\partial_{g}}{k_{i}}$ and $\beta_{i} \gamma_{i} \equiv 1 \bmod k_{i}$

\section{System Model and Security Requirements}

\subsection{System Model}

The system model of our proposed scheme is illustrated in Fig. 1. There are three main components: a TA, OBUs and RSUs.

TA: Generally, TA is considered as a highly trusted and powerful component in the proposed authentication scheme. Moreover, TA may generate and distribute group key for vehicles for secure V2V communications. Once emergencies happen, TA may track the malicious vehicles with the vehicle's pseudonym[12][14].

RSU: RSUs are fixed infrastructures deployed on 


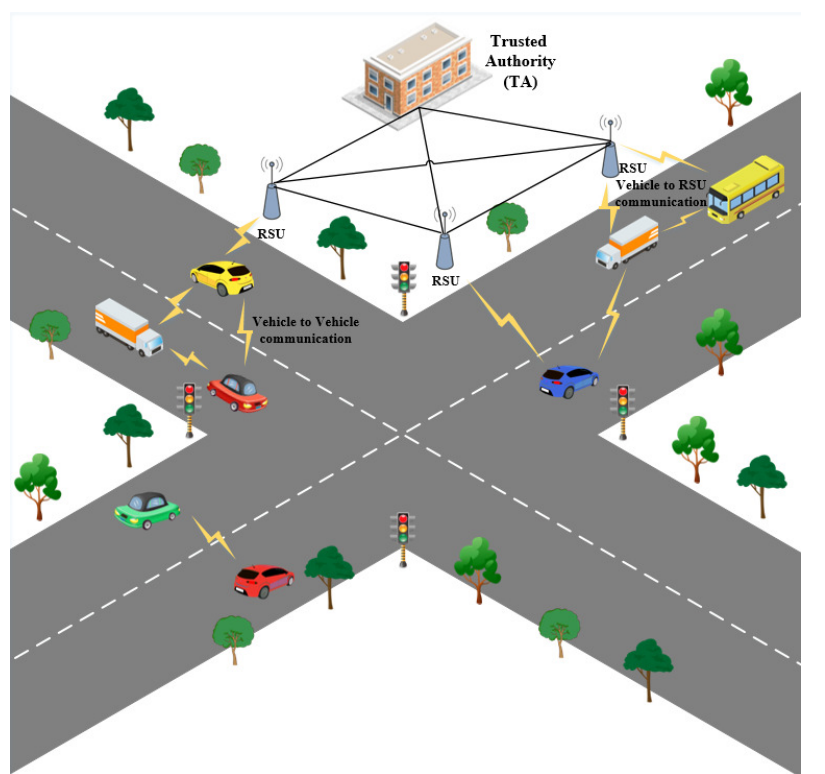

Fig. 1 System model

the roadside or some installations. RSU is not completely trusted. Therefore, it must be authenticated by vehicles. In the proposed scheme, they are relay nodes between vehicles and TA[12][14].

OBU: Each vehicle is equipped with an on-board unit (OBU) with tamper-proof equipment. The OBU is responsible for storing the real identity of the vehicle, synchronizing the clock and some secret information to perform cryptographic operations[12][14].

\subsection{Security Requirements}

Our scheme should satisfy following security.

1) Message authentication: Vehicle must be able to check the validity of the message before receiving it to protect it from false message attack.

2) Conditional privacy of the vehicles: The vehicle's real identity cannot be obtained by the adversary, but TA can get the real identity if a vehicle sends a malicious message.

3) Forward and backward security of group-key agreement: When a vehicle leaves the group, the group key will be updated, and the left vehicle cannot compute the new group key. When a vehicle joins the group, if it wants to get previous information, it needs the previous group key. Otherwise it cannot compute the previous key.

4) Resistance to replay attack: The adversary captures the intermediate security transfer information and repeats them for unauthorized access to the security key or message.

5) Resistance to modification attack: The adversary
Table 1 Notations

\begin{tabular}{ll}
\hline Notations & Descriptions \\
\hline$r$ & A positive integer \\
$p$ & An odd prime number \\
$q$ & A large prime number used to select ran- \\
$G F(q)$ & dom numbers, keys, etc. \\
$Z$ & A finite group \\
$f(y)$ & A finite field \\
$T A$ & An odd prime number \\
$R_{j}$ & The trusted authority \\
$V_{i}$ & The jth road-side-unit \\
$S K_{R_{j}}$ & The ith vehicle \\
$\left(x_{R_{j}}, T_{S K_{R_{j}}}\left(x_{R_{j}}\right)\right)$ Public key of $R_{j}$ \\
$s s$ \\
$s k_{r_{j}}$ & System private key selected by TA \\
$I D_{R_{j}}$ & Secret key shared between the $R_{j}$ and \\
$I D_{V_{i}}$ & the TA \\
$P I D_{V_{i}}$ & Real identity of $R S U_{j}$ \\
$U S K_{i}$ & Real identity of $V_{i}$ \\
$H(\cdot)$ & The pseudonym used in the communica- \\
$T_{s}(\cdot)$ & tion process of vehicle $V_{i}$ \\
& The group key generation key for each \\
& OBU $U_{j}$ \\
& A secure cryptographic hash function \\
& The Chebyshev polynomial
\end{tabular}

may modify, delete, or change a specific part of the message and broadcast the modified message to achieve some selfish purposes.

\section{Proposed Scheme}

In this section, we present our proposed scheme. We list frequently used notations in Table 1, and the overall flow chart of the scheme is briefly described in Fig. 2. We use Chebyshev chaotic map to do authentication when vehicles join the range of a RSU and provide a key agreement scheme. The detailed process of the proposed scheme will be described as follows.

\subsection{System Initialization phase}

Prior to the deployment of the VANET, TA needs to generate some public and private parameters for the system. These parameters are preloaded into the OBU of the vehicle at vehicle registration and sent to the $\mathrm{RSU}$ at RSU registration. This phase is described below.

1) TA choose a positive integer $r$ and an odd prime number $p$, then compute $q=p^{r}$, we assume that computations over $G F(q)$ are carried out modulo an irreducible polynomial $f(y)[7]$.

2) TA selects a random number $s \in Z$, where $s \neq 0,1$, as system private key.

3) TA selects a secure cryptographic hash function 


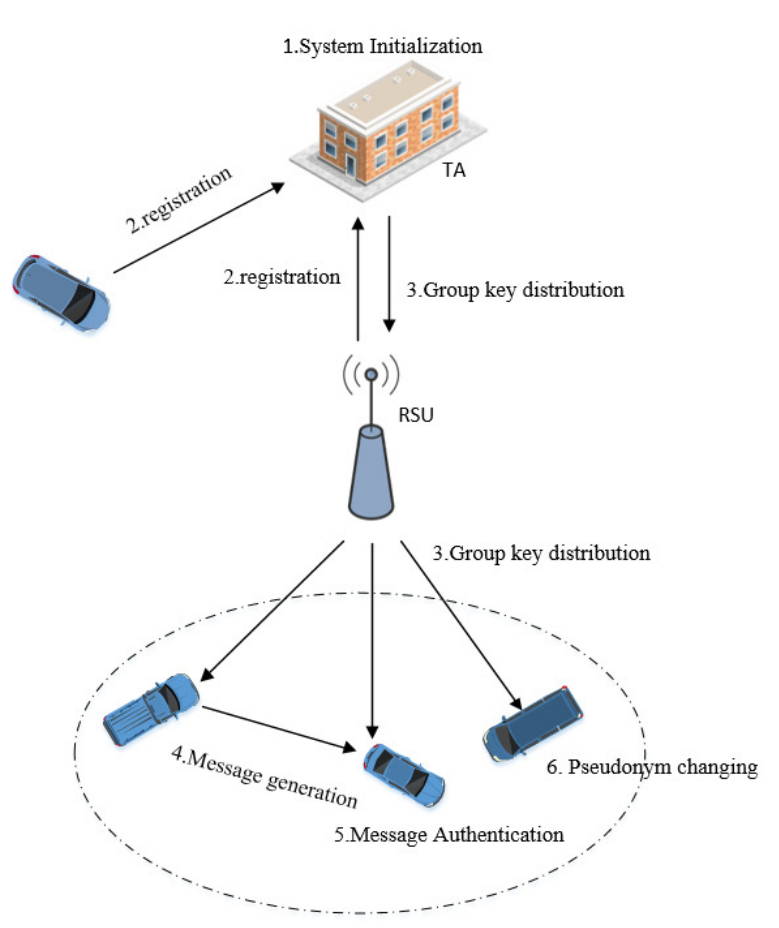

Fig. 2 The system model of VANET

$H$, where $H:\{0,1\}^{*} \rightarrow\{0,1\}, H$ denotes the chaotic Hash function[15].

4) TA choose random number $x, x \in G F(q)$, $x \neq 0,1$, compute $T_{s}(x)$.

5) TA share $\left\{T_{s}(x), x, H\right\}$ with all vehicles and RSUs during the registration of vehicles and RSUs, while keeping $s$ as its secret key.

\subsection{Offline Registration phase}

In this stage, vehicles and RSUs need to provide the essential information like name, address, phone number, email id etc. to TA to make offline registration.

\section{Registration of $O B U$.}

1) Vehicle $V_{i}$ first approaches the TA office directly to make offline registration and provide the essential information such as name, address, phone number and email to the TA.

2) After completing the registration process, the TA provides the generation key of group key $U S K_{i}$ for each $V_{i}$ and store $\left\{I D_{V_{i}}, U S K_{i}\right\}$ in its tracking list database.

\section{Registration of $R S U$.}

1) RSU $R_{j}$ sends privacy information (such as identity $I D_{R_{j}}$ and location information) to TA.

2) After checking the legitimacy of $R_{j}$, TA selects an integer $S K_{R_{j}} \in Z, S K_{R_{j}} \neq 0,1$ and selects a random number $x_{R_{j}} \in G F(q), x_{R_{j}} \neq 0,1$ and computes

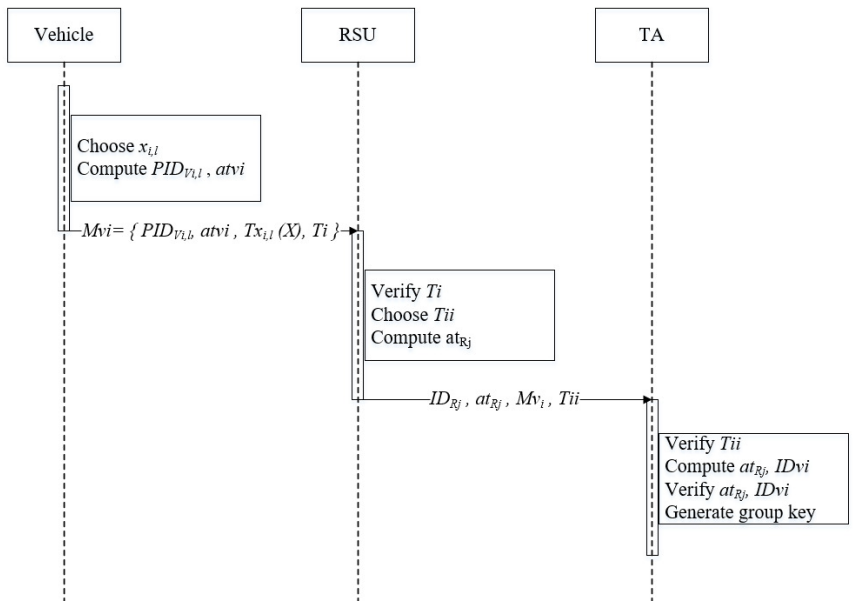

Fig. 3 Vehicle authentication protocol

$T_{S K_{R_{j}}}\left(x_{R_{j}}\right)$, where the $S K_{R_{j}}$ means the secret key of $R_{j}$ and $\left(x_{R_{j}}, T_{S K_{R_{j}}}\left(x_{R_{j}}\right)\right)$ represents the public key of $R_{j}$.

3) TA assign $R_{j}$ a secret key $s k_{r_{j}}$ through a secure channel, where $s k_{r_{j}}$ is shared by $R_{j}$ and TA.

4) TA store $\left\{I D_{R_{j}}, s k_{r_{j}}\right\}$ in its RSU list.

\subsection{Group key distribution phase}

In order to get a group key for message generation and authentication within the same RSU range, users must be authenticated to ensure the reliability of group key. The process is described as follows.

Authentication message generation The process of authentication phase is shown in Fig.3

(1) Vehicles send authentication message to TA when joining in the group within the range of $R_{j}$.

(2) $V_{i}$ chooses random number $x_{i, l}, \quad l=$ $1,2,3 \ldots \ldots k$, and publishes $T_{x_{i, l}}(x) . V_{i}$ generates pseudonym $P I D_{V_{i, l}}=I D_{V_{i}} T_{s x_{i, l}}(x)(\bmod f(y)) . V_{i}$ selects the current timestamp $T_{i}$, and calculates $a t_{V_{i}}=H\left(I D_{V_{i}} \| T_{i}\right)$.

(3) $V_{i}$ obtains $R_{j}$ 's authentic public key $\left(x_{R_{j}}, T_{S K_{R_{j}}}\left(x_{R_{j}}\right)\right)$ and represents the message as $M_{v_{i}}=\left\{P I D_{V_{i, l}}, a t_{V_{i}}, T_{x_{i, l}}(x), T_{i}\right\}$. Then it selects a random number $r \in Z, r \neq 0,1$, computes $T_{r}\left(x_{R_{j}}\right)$, $T_{r S K_{R_{j}}}\left(x_{R_{j}}\right)=T_{r}\left(T_{S K_{R_{j}}}\left(x_{R_{j}}\right)\right)$ and sends the cipher text $C=\left(T_{r}\left(x_{R_{j}}\right), M_{v_{i}} \cdot T_{r} S K_{R_{j}}\left(x_{R_{j}}\right)\right)$ to $R_{j}$ through an open channel.

(4) $R_{j}$ decrypts the message with its secret key $S K_{R_{j}}$ and gets the message from vehicle $V_{i}, R_{j}$ checks the validity of timestamp.

(5) $R_{j}$ selects the current timestamp $T_{i i}$, computes $a t_{R_{j}}=H\left(M_{v_{i}}\left\|s k_{r_{j}}\right\| T_{i i}\right)$. 


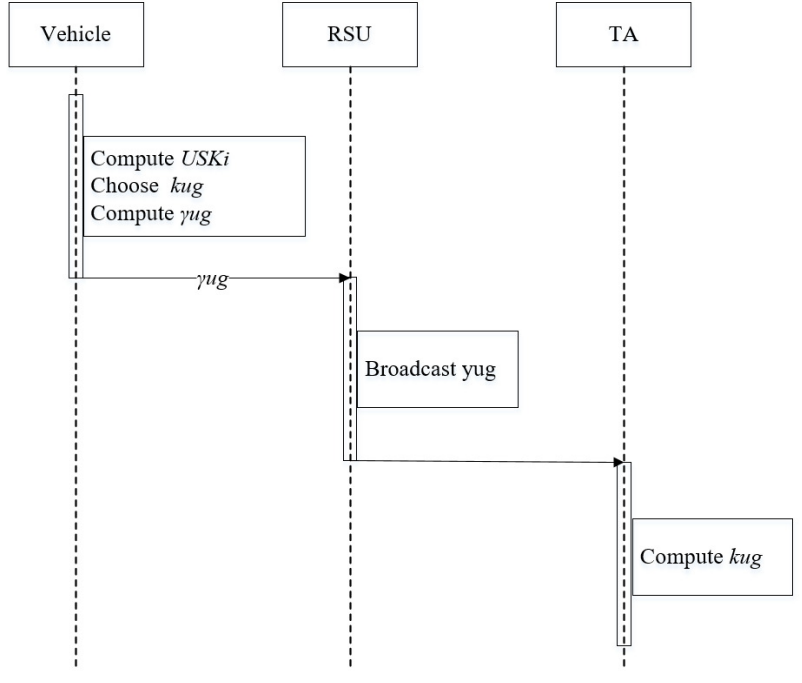

Fig. 4 Group key distribution protocol

(6) $R_{j}$ sends the message $\left\{I D_{R_{j}}, a t_{R_{j}}, M_{v_{i}}, T_{i i}\right\}$ to TA via a secure channel.

Authentication message verification In this phase, TA validates the received authentication message through $R_{j}$.

(1)TA verifies the timestamp $T_{i i}$ when it receives the message $\left\{I D_{R_{j}}, a t_{R_{j}}, M_{v_{i}}, T_{i i}\right\}$.

(2)TA calculates $a t_{R_{j}}^{\prime}=H\left(M_{v_{i}}\left\|s k_{r_{j}}\right\| T_{i i}\right)$. If $a t_{R_{j}}^{\prime}=a t_{R_{j}} \cdot R_{j}$ is a legitimate RSU.

(3) TA computes $I D_{V_{i}}=$ $P I D_{V_{i, l}} T_{s x_{i, l}}(X)^{-1}(\bmod f(y)), a t_{V_{i}}^{\prime}=H\left(I D_{V_{i}} \| T_{i}\right)$, if $a t_{V_{i}}^{\prime}=a t_{V_{i}}$, then check if $I D_{V_{i}}$ is in the tracking list, the vehicle is legal. TA get $U S K_{i}$ to compute group key.

Group key distribution In order to ensure the communication within the same RSU range, vehicles must obtain a group key for message generation and verification. In this phase, a mutual authentication process must be conducted to ensure the reliability of group key, as shown in Fig 4. The process is described as follows.

1) When $V_{i}$ enters the range of $R_{j}, R_{j}$ receives $V_{i}$ 's broadcast with $P I D_{V_{i, l}}$, then $R_{j}$ transmits the message to TA. TA can compute $I D_{V_{i}}$, and get $U S K_{i}$ by tracking list $\left\{I D_{V i}, U S K_{i}\right\}$.

2) TA multiplies all $U S K_{i}$ in the range of $R_{j}$, $i=1,2,3, \ldots, n, \partial g=\prod_{i=1}^{n} U S K_{i}$. It then computes

$x_{i}=\frac{\partial g}{U S K_{i}}$, where $i=1,2,3, \ldots, n$

$x_{i} \times y_{i} \equiv 1 \bmod U S K_{i}$

$\operatorname{var}_{i}=x_{i} \times y_{i}$

$\mu=\sum_{i}^{n} \operatorname{var}_{i}$.

3) $V_{i}$ gets the group secret key from the TA via $R_{j}$.

a) TA chooses a random number $k u g$ as the group secret key for vehicles in the range of $R_{j}$.

b) TA computes $y u g=k u g \times \mu$, and timestamp $T_{i i i}$ , and passes it to $R_{j}$.

c) $R_{j}$ broadcast $y u g$ and $T_{i i i}$.

d) $V_{i}$ can get $k u g$ by a module division operation: yug $\bmod U S K_{i}=k u g$.

\section{Group key updating .}

When a vehicle leaves the range of RSU, the following steps are performed.

a) TA compute $\mu^{\prime}=\mu-\operatorname{var}_{i}$ and choose a new group key $k u g^{\prime}$.

b) Vehicles in the group update the group key by performing group key computation of the group key distribution phase.

When a vehicle enters the group, the following steps are performed:

a) TA authenticate the vehicle by the authentication process as mentioned in Section $\mathrm{C}$ authentication process. If authentication fails, the vehicle cannot join the group. Otherwise, process the next step.

b) TA computes $\mu^{\prime}=\mu+v_{a r}$ and chooses a new group key $k u g^{\prime}$.

c) Vehicles in the group update the group key by performing group key computation of the group key distribution phase.

\subsection{Message generation phase}

1) $V_{i}$ gathers the road condition and produces message $\mathrm{M}$ and time stamp $T_{v_{i}}$.

2) $V_{i}$ chooses one $P I D_{i}$, then compute $M V=$ $H_{k u g}\left(P I D_{i}, M, T_{v_{i}}\right)$.

3) $V_{i}$ broadcast message $\left\{P I D_{i}, M, T_{v_{i}}, M V\right\}$.

\subsection{Message authentication phase}

1) Once receiving a message, receiver $V_{j}$ firstly compares the time stamp $T_{v_{i}}$ in the message with the current time stamp $T_{i}^{\prime}$. If $\left(T_{i}^{\prime}-T_{v_{i}}\right) \leq \Delta t$ holds, where $\Delta t$ means permissible time delay for message transmission, the message is valid. Otherwise, the message will be abandoned.

2) Using group key to compute $M V^{\prime}=$ $H_{k u g}\left(P I D_{i}, M, T_{v_{i}}\right)$, If $M V^{\prime}=M V$, both vehicle and message are authenticated successfully. Otherwise, the message may has been modified. In this case, receiver may send the message to TA and report the situation. The process is illustrated in Fig. 5. 


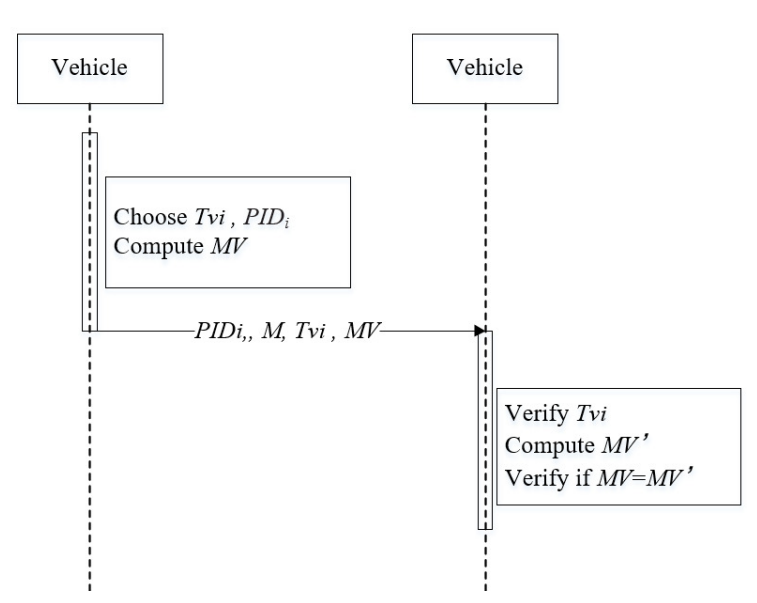

Fig. 5 Message authentication protocol

\subsection{Pseudonym changing}

When a vehicle reaches the social spot, it can change its pseudonym $P I D_{V_{i, l}}$ to protect its location privacy. Vehicle can choose random number $x_{i, l}, l=$ $1,2,3 \ldots \ldots k$ and publish $T_{x_{i, l}(x)}$, then generate a different pseudonym

$P I D_{V_{i, l}}=I D_{V_{i}} T_{s x_{i, l}}(x)(\bmod f(y))$

\section{Security Analysis}

A secure conditional privacy-preserving authentication scheme for VANET should be able to withstand various attacks mentioned in Section IV. In this section, we firstly demonstrate that our scheme achieves security goals by providing the authentication proof based on BAN Logic [2]. Then we state that our scheme satisfies security requirements by informal security discussion.

\subsection{Formal security proof}

In this subsection, we use BAN logic [2] to formally analyze our scheme. Fundamental rules for BAN logic are listed as follows.

- R1: Message-meaning rule: $\frac{P \mid \equiv P \leftrightarrow Q, P \triangleleft\langle X\rangle_{k}}{P|\equiv Q| \sim X}$.

- R2: Nonce-verification rule: $\frac{P|\equiv \#(X), P| \equiv Q \mid \sim X}{P|\equiv Q| \equiv X}$.

- R3: Jurisdiction rule: $\frac{P|\equiv Q \Rightarrow X, P| \equiv Q \mid \equiv X}{P \mid \equiv X}$.

- R4: Freshness-conjuncatenation rule: $\frac{P \mid \equiv \#(X)}{P \mid \equiv \#(X, Y)}$.

- R5: Session-key rule: $\frac{P|\equiv \# X, P| \equiv Q \mid \equiv X}{P \mid \equiv P \stackrel{K}{\longleftrightarrow} Q}$.

There are four goals need to be proved:

- Goal 1: TA $\equiv I D_{R_{j}}, a t_{R_{j}}, M_{v_{i}}, T_{i i}$.

- Goal 2: $R_{j} \mid \equiv P I D_{V_{i, l}}, a t_{V_{i}}, T_{x_{i, l}}(x), T_{i}$.

- Goal 3: $V_{i} \mid \equiv k u g$.
- Goal 4: $V_{j}\left|\equiv V_{i}\right| \equiv P I D_{i}, M, T_{v_{i}}, M V$.

For the formal analysis, the message exchanged among $V_{i}, R_{j}$, and $T A$ are idealized as follows.

- M1: $V_{i} \rightarrow R_{j}:\left\{P I D_{V_{i, l}}, a t_{V_{i}}, T_{x_{i, l}}(x), T_{i}\right\}$

- M2: $R_{j} \rightarrow T A:\left\{I D_{R_{j}}, a t_{R_{j}}, M_{v_{i}}, T_{i i}\right\}$

- M3: $T A \rightarrow V_{i}:$ yug

- M4: $V_{i} \rightarrow V_{j}:\left\{P I D_{i}, M, T_{v_{i}}, M V\right\}$

Prerequisites for the formal proof are as follows.

- $\mathrm{A} 1: R j \mid \equiv R j \stackrel{\left(x_{R_{j}}, T_{S K_{R_{j}}}\left(x_{R_{j}}\right)\right)}{\longleftrightarrow} V_{i}$

- $\mathrm{A} 2: R_{j} \mid \equiv \# T_{i}$

- A3: $R_{j} \mid \equiv V_{i} \Rightarrow P I D_{V_{i, l}}, a t_{V_{j}}, T_{x_{i, l}}(X), T_{i}$

- A4: $T A \mid \equiv \# T_{i i}$

- $\mathrm{A} 5: T A \mid \equiv R_{j} \Rightarrow\left(I D_{R_{j}}, s k_{r_{j}}\right)$

- $\mathrm{A} 6: V_{i} \mid \equiv V_{i} \stackrel{U S K i}{\longleftrightarrow} T A$

- A7: $V_{i} \mid \equiv \# T_{i i i}$

- A8: $V_{i}|\equiv T A| \equiv U S K_{i}$

- A9: $V_{j} \mid \equiv V_{j} \stackrel{k u g}{\longleftrightarrow} V_{i}$

- A10: $V_{j} \mid \equiv \# T_{v_{i}}$

Based on the aforementioned assumptions and logical postulates of BAN logic, we provide formal proof of our proposed scheme as follows.

By message 1, the following statement is obtained:

- S1: $R j \triangleleft\left\{P I D_{V_{i, l}}, a t_{V_{j}}, T_{x_{i, l}}(x), T_{i}\right\}_{\left(x_{R_{j}}, T_{S K_{R_{j}}}\left(x_{R_{j}}\right)\right)}$ According to S1, A1 and R1, we have:

- S2: $R_{j}\left|\equiv V_{i}\right| \sim P I D_{V_{i, l}}, a t_{V_{i}}, T_{x_{i, l}}(x), T_{i}$ According to S2, A2, R2 and R4, we have

- S3: $R_{j}|\equiv V i| \equiv P I D_{V_{i, l}}, a t_{V_{i}}, T_{x_{i, l}}(x), T_{i}$

According to S3, A3 and R3. we have

- $\mathrm{S} 4: R_{j} \mid \equiv P I D_{V_{i, l}}, a t_{V_{i}}, T_{x_{i, l}}(x), T_{i}$

(Goal 2)

By message 2, the following statement is obtained

- $\mathrm{S} 5: T A\left|\equiv R_{j}\right| \sim I D_{R_{j}}, a t_{R_{j}}, M_{v_{i}}, T_{i i}$

According to S5, A4, R2 and R4, we have:

- S6: $T A\left|\equiv R_{j}\right| \equiv I D_{R_{j}}, a t_{R_{j}}, M_{v_{i}}, T_{i i}$

According to S6, A5 and R3, we have:

- S7: $T A \mid \equiv I D_{R_{j}}, a t_{R_{j}}, M_{v_{i}}, T_{i i}$

(Goal 1)

By message 3

- $\mathrm{S} 8: V_{i} \triangleleft k u g \times \mu$

According to S8, A6 and R1, we have:

- S9: $V_{i}|\equiv T A| \sim k u g \times \mu$

According to S9, A7, R2 and R4, we have

- $\mathrm{S} 10: V_{i}|\equiv T A| \equiv k u g \times \mu$

According to S10, A8, R3, we have:

- $\mathrm{S} 11: V_{i} \mid \equiv k u g$

(Goal 3)

By message 4

- $\mathrm{S} 12: V_{j} \triangleleft P I D_{i}, M, T_{v_{i}}, M V$

By S12,A9 and R1:

- $\mathrm{S} 13: V_{j}\left|\equiv V_{i}\right| \sim P I D_{i}, M, T_{v_{i}}, M V$

Based on S13, A10, R2, R4, the following equation can be gotten.

- S14: $V_{j}\left|\equiv V_{i}\right| \equiv P I D_{i}, M, T_{v_{i}}, M V$

(Goal 4)

From the above-mentioned analysis, we can see our protocol achieves all the goals $1-4$, which collectively 
guarantee the mutual authentication between nodes. Besides, it shows that vehicles can get correct group key after a mutual authentication process and vehicles within the same RSU range with same group key can communicate with each other securely.

\subsection{Informal security analysis}

1) Message authentication

A vehicle must be authenticated before it gets group key to communicate with other vehicles. The sender generates a Hash value $M V=H_{k u g}\left(P I D_{i}, M, T_{v_{i}}\right)$. The receiver will check the validity of Hash by compute $M V^{\prime}$ using group key. Only when $M V^{\prime}=M V$ the message will be accepted.

2) Conditional privacy

Preservation of the vehicle's identity: In our scheme, vehicles communicate with each other using $P I D_{V_{i, l}}$, so it can protect the real identity of the vehicle in an open communication channel.

Location privacy: Our scheme provides pseudonym changing mechanism, vehicles change their PID when reaching social spots, which protects location privacy.

Traceability: If there is a malicious vehicle, TA can get the vehicle's real identity by computing $I D_{V_{i}}=$ $P I D_{V_{i, l}} T_{s x_{i, l}}(x)(\bmod f(y))$.

3) Forward and backward security

When a vehicle leaves the group, the group key will be updated, and the vehicle which leaves the group is impossible to compute the new group key. Therefore, our scheme satisfies the forward security. When a vehicle joins the group, if it wants to get previous information, it needs the previous group key. Because the newly joined vehicle cannot compute the previous key, it satisfies the backward security.

4) Replay attack

In a replay attack, malicious users repeat the previously received messages. In our scheme, we use timestamp in each message so that receivers can check the freshness of authentication messages by checking whether $T_{v_{i}}^{\prime}-T_{v_{i}} \leq \Delta t$ holds.

5) Modification attack

An adversary may modify a message and rebroadcast it. However, we use keyed hash function $\mathrm{H}$ to authenticate messages. Without the group key kug, it's difficult to generate a valid Hash. Any modification of message $\left\{P I D_{i}, M, T_{v_{i}}, M V\right\}$ will cause $M V \neq$ $H_{k u g}\left(P I D_{i}, M, T_{v_{i}}\right)$. The message can't be authenticated and accepted. Therefore, our scheme can resist modification attack.

\section{Perfomance Analysis}

In this section, we perform a performance analysis of our scheme from both computational overhead and communication overhead, and compare it with existing schemes. Our implementation is performed on a laptop consists of an Intel Core i5-8400 CPU@2.80 GHz, 8G RAM, and Windows 10 OS. Some notations about execution time are defined in table 2 :

\subsection{Computation Cost and Comparison}

To give an overall analysis of computational cost, we will compute the time overhead of the following process: user authentication, group key distribution, message generation and message authentication. The execution time of related cryptographic operations are listed in Table 3 .

In our scheme, for a vehicle to generate a message, 4 Chebyshev encryption $4 T_{c h}, 2$ hash function $2 T_{h}$ and a keyed hash function operation using chaos map $T_{H_{k e y}}$ are required. Similarly, for a vehicle to authenticate a message, 4 Chebyshev encryption $4 T_{c h}, 2$ hash function $2 T_{h}$ and a keyed hash function operation using chaos map $T_{H_{k e y}}$ are required.

Table 4 lists the comparison of the computation cost between several related schemes and our proposed scheme. It can be seen from Table 4 that the computational cost of the proposed scheme is superior to other schemes.

\subsection{Communication Overhead}

In this part, we compare the communication overhead of the proposed scheme with several existing schemes.

We assume that the sizes of the elements in $G_{1}$ and $G$ are 128 bytes and 40 bytes, respectively. In addition, let the output of a hash function and the size of the time stamp are 20 bytes and 4 bytes, respectively. Moreover, we assume that the origin messages are included in the finite field $Z_{p}^{*}$ and have a size of 20 bytes.

We focus on the analysis of the communication overhead in the following three processes: authentication message generation, authentication message verification, and group-key generation. The communication overhead of several schemes is listed in Table 5.

In the scheme proposed by He et al. [4] the authentication message is $\left\{M_{i}, A I D_{i}, T_{i}, R_{i}, \sigma_{i}\right\}$, where $A I D_{i}=\left\{A I D_{i}^{1}, A I D_{i}^{2}\right\},\left\{A I D_{i}^{1}, A I D_{i}^{2}, R_{i}\right\} \in G, \sigma_{i} \in$ $Z_{q}^{*}, T_{i}$ is the timestamp, so the size of the authentication message is $40 \times 3+20 \times 2+4=164$ bytes. 
Table 2 Notations

\begin{tabular}{ll}
\hline Notations & Descriptions \\
\hline$T_{b p}$ & the execution time of one bilinear pairing operation $\hat{e}(\hat{U}, \hat{V})$, where $U, V \in G 1$ \\
$T_{e m}$ & the execution time of one scale multiplication operation on ECC \\
$T_{e a}$ & the execution time of one point addition operation on ECC \\
$T_{e p}$ & the execution time of one exponentiation operation on point \\
$T_{h}$ & the execution time of one hash function operation using SHA-1 \\
$T_{c h}$ & the execution time of chebyshev' encryption \\
$T_{H_{k e y}}$ & the execution time of one keyed hash function operation using chaos map \\
\hline
\end{tabular}

Table 3 Execution time of several cryptographic operations

\begin{tabular}{ll}
\hline Cryptographic operation & Execution time (millisecond) \\
\hline$T_{b p}$ & $14.69 \mathrm{~ms}$ \\
$T_{e m}$ & $0.715 \mathrm{~ms}$ \\
$T_{e a}$ & $0.073 \mathrm{~ms}$ \\
$T_{e p}$ & $8.12 \mathrm{~ms}$ \\
$T_{h}$ & $0.045 \mathrm{~ms}$ \\
$T_{c h}$ & $0.34 \mathrm{~ms}$ \\
$T_{H_{\text {key }}}$ & $0.061 \mathrm{~ms}$ \\
\hline
\end{tabular}

In the scheme proposed by Azees et al. [1] vehicles send messages as $\left(M \|\right.$ sig $\left\|Y_{k}\right\|$ Cert $\left._{k}\right)$, where $\operatorname{Cert}_{k}=\left\{Y_{k}\left\|E_{i}\right\| D I D_{u_{i}}\left\|\gamma_{u}\right\| \gamma_{v}\|c\| \lambda\left\|\sigma_{1}\right\| \sigma_{2}\right\}$ $\left\{\right.$ sig, $\left.Y_{k}, E_{i}, D I D_{u_{i}}, \gamma_{u}, \gamma_{v}\right\} \in G 1,\left\{M, \lambda, \sigma_{1}, \sigma_{2}\right\} \in Z_{q}^{*}$, $\mathrm{c}$ is a hash operation result. Therefore, the communication overhead is $7 \times 128+5 \times 20=996$ bytes.

The authentication message in the scheme of Lo et al. [9] is $\left\{P I D_{i, k}, M_{i}, t t_{i}, K_{i}, R_{i}, V_{i}\right\}$, where $P I D_{i, k}=\left\{A I D_{i, 1}, A I D_{i, 2}\right\},\left\{A I D_{i, 1}, K_{i}, R_{i}\right\} \in G$ and $\left\{A I D_{i, 2}, V_{i}\right\} \in Z_{q}^{*}$. Hence, the communication overhead is $40 \times 3+20 \times 3+4=184$ bytes.

In our scheme, the authentication message is $\left\{P I D_{i}, M, T_{i}, M V\right\}, \quad M V=H_{k u g}\left(P I D_{i}, M, T_{i}\right)$. Therefore, the total communication overhead is $40+$ $20+20+4=84$ bytes. We can see that the overall communication overhead of our solution is relatively low.

\section{Conclusion}

In this paper, we have proposed a high-efficient authentication and group-key agreement scheme for VANET. The proposed scheme uses Chebyshev chaotic mapping instead of the time-consuming elliptic curve and bilinear pairing technique to do authentication, which increases the efficiency. While ensuring security, the overall computation and communication overhead are reduced. Moreover, our solution includes a group key distribution scheme that allows group members to access the group key while providing forward and backward security. Meanwhile, location privacy is provided by adopting pseudonym changing at social spot strategy.

\section{Declarations}

Conflicts of interest The authors declare that they have no conflict of interest.

Data Availability All data generated or analysed during this study are included in this published article.

\section{References}

1. Azees, M., Vijayakumar, P., Deboarh, L.J.: Eaap: Efficient anonymous authentication with conditional privacy-preserving scheme for vehicular ad hoc networks. IEEE Transactions on Intelligent Transportation Systems 18(9), 2467-2476 (2017)

2. Burrows, M., Abadi, M., Needham, R.M.: A logic of authentication. Proceedings of the Royal Society of London. A. Mathematical and Physical Sciences 426(1871), 233-271 (1989)

3. Commission, F.C., et al.: Amendment of the commission's rules regarding dedicated short-range communication service in the 5.850-5.925 ghz band. FCC, Washington, DC, USA, Tech. Rep. FCC pp. 02-302 (2002)

4. He, D., Zeadally, S., Xu, B., Huang, X.: An efficient identity-based conditional privacy-preserving authentication scheme for vehicular ad hoc networks. IEEE Transactions on Information Forensics and Security 10(12), 2681-2691 (2015)

5. Jiang, D., Delgrossi, L.: Ieee 802.11 p: Towards an international standard for wireless access in vehicular environments. In: VTC Spring 2008-IEEE Vehicular Technology Conference, pp. 2036-2040. IEEE (2008)

6. Li, X., Liu, Y., Yin, X.: An anonymous conditional privacy-preserving authentication scheme for vanets. In: 2019 IEEE 21st International Conference on High Performance Computing and Communications; IEEE 17th International 
Table 4 Computation cost of different schemes

\begin{tabular}{llll}
\hline Schemes & message generation & message verification & Total execution time $(\mathrm{ms})$ \\
\hline He et al & $3 T_{e m}+3 T_{h}$ & $3 T_{e m}+2 T_{e a}+2 T_{h}$ & 4.661 \\
Azee et al & $T_{e p}+T_{h}$ & $2 T_{b p}+5 T_{e p}+2 T_{e a}$ & 78.291 \\
Lo et al & $2 T_{e m}+2 T_{h}$ & $3 T_{e m}+2 T_{e a}+2 T_{h}$ & 3.901 \\
Our scheme & $2 T_{h}+4 T_{c h}+H_{\text {key }}$ & $2 T_{h}+4 T_{c h}+H_{\text {key }}$ & 3.022 \\
\hline
\end{tabular}

Table 5 Communication overhead of different schemes

\begin{tabular}{ll}
\hline Schemes & Communication overhead \\
\hline He et al & 164 bytes \\
Azee et al & 996 bytes \\
Lo et al & 184 bytes \\
Our scheme & 84 bytes \\
\hline
\end{tabular}

Conference on Smart City; IEEE 5th International Conference on Data Science and Systems (HPCC/SmartCity/DSS), pp. 1763-1770. IEEE (2019)

7. Lima, J.B., Panario, D., de Souza, R.C.: Public-key encryption based on chebyshev polynomials over gf (q). Information Processing Letters 111(2), 51-56 (2010)

8. Liu, Y., Wang, L., Chen, H.H.: Message authentication using proxy vehicles in vehicular ad hoc networks. IEEE Transactions on vehicular technology 64(8), 3697-3710 (2014)

9. Lo, N.W., Tsai, J.L.: An efficient conditional privacy-preserving authentication scheme for vehicular sensor networks without pairings. IEEE Transactions on Intelligent Transportation Systems 17(5), 1319-1328 (2015)

10. Raya, M., Hubaux, J.P.: Securing vehicular ad hoc networks. Journal of computer security 15(1), 39$68(2007)$

11. Shen, X., Cheng, X., Yang, L., Zhang, R., Jiao, B.: Data dissemination in vanets: A scheduling approach. IEEE Transactions on Intelligent Transportation Systems 15(5), 2213-2223 (2014)

12. Vijayakumar, P., Azees, M., Chang, V., Deborah, J., Balusamy, B.: Computationally efficient privacy preserving authentication and key distribution techniques for vehicular ad hoc networks. cluster computing 20(3), 2439-2450 (2017)

13. Vijayakumar, P., Azees, M., Kannan, A., Deborah, L.J.: Dual authentication and key management techniques for secure data transmission in vehicular ad hoc networks. IEEE Transactions on Intelligent Transportation Systems 17(4), 1015-1028 (2015)

14. Vijayakumar, P., Chang, V., Deborah, L.J., Balusamy, B., Shynu, P.: Computationally efficient privacy preserving anonymous mutual and batch authentication schemes for vehicular ad hoc networks. Future generation computer systems $\mathbf{7 8}$, 943-955 (2018)

15. Wang, Y., Liao, X., Xiao, D., Wong, K.W.: Oneway hash function construction based on $2 \mathrm{~d}$ coupled map lattices. Information Sciences 178(5), 1391-1406 (2008)

16. Zhang, C., Lu, R., Lin, X., Ho, P.H., Shen, X.: An efficient identity-based batch verification scheme for vehicular sensor networks. In: IEEE INFOCOM 2008-The 27th Conference on Computer Communications, pp. 246-250. IEEE (2008)

17. Zhang, L.: Cryptanalysis of the public key encryption based on multiple chaotic systems. Chaos, Solitons \& Fractals 37(3), 669-674 (2008)

18. Zheng, X., Huang, C.T., Matthews, M.: Chinese remainder theorem based group key management. In: Proceedings of the 45th annual southeast regional conference, pp. 266-271 (2007) 
Figures

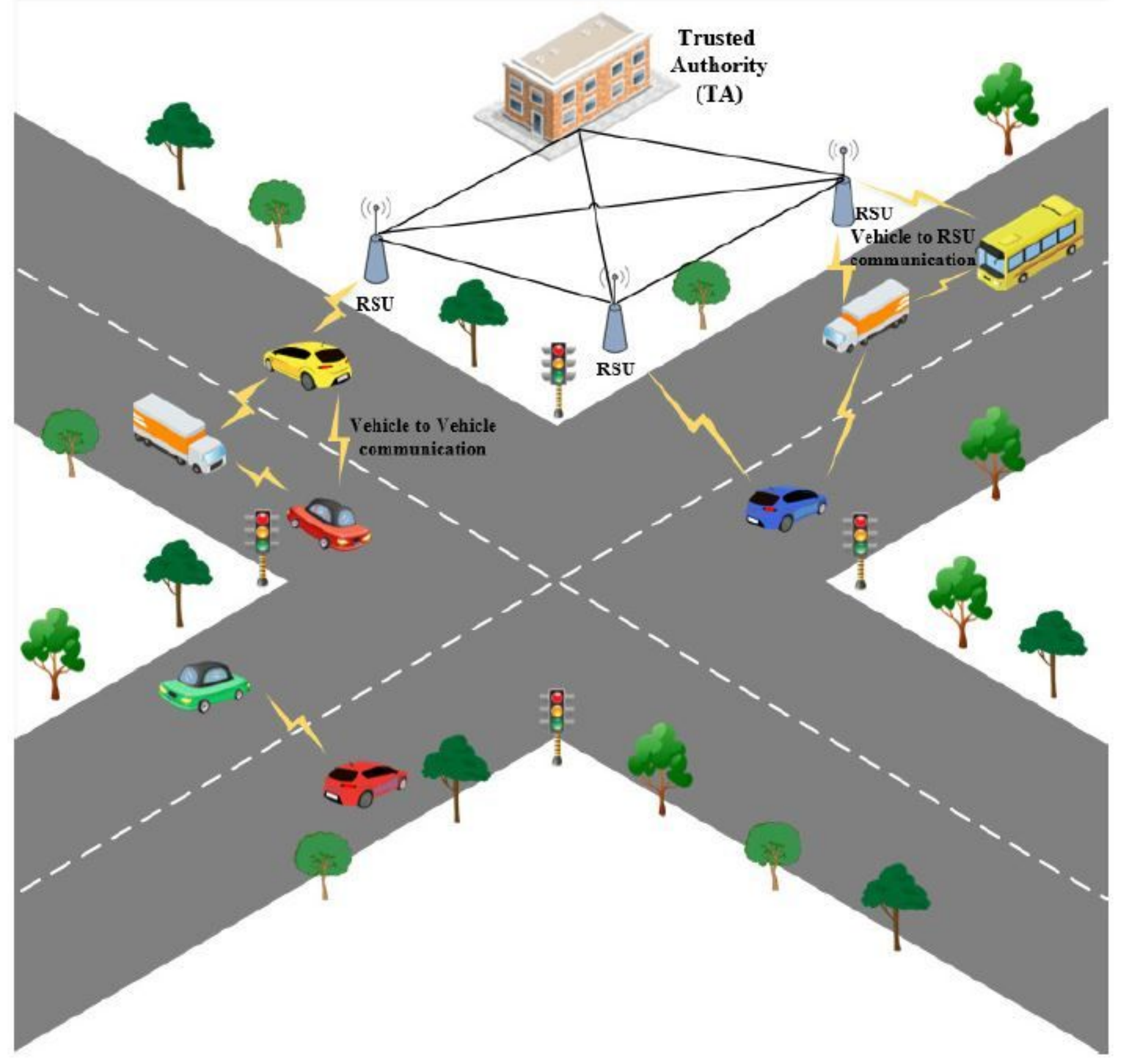

Figure 1

System model 


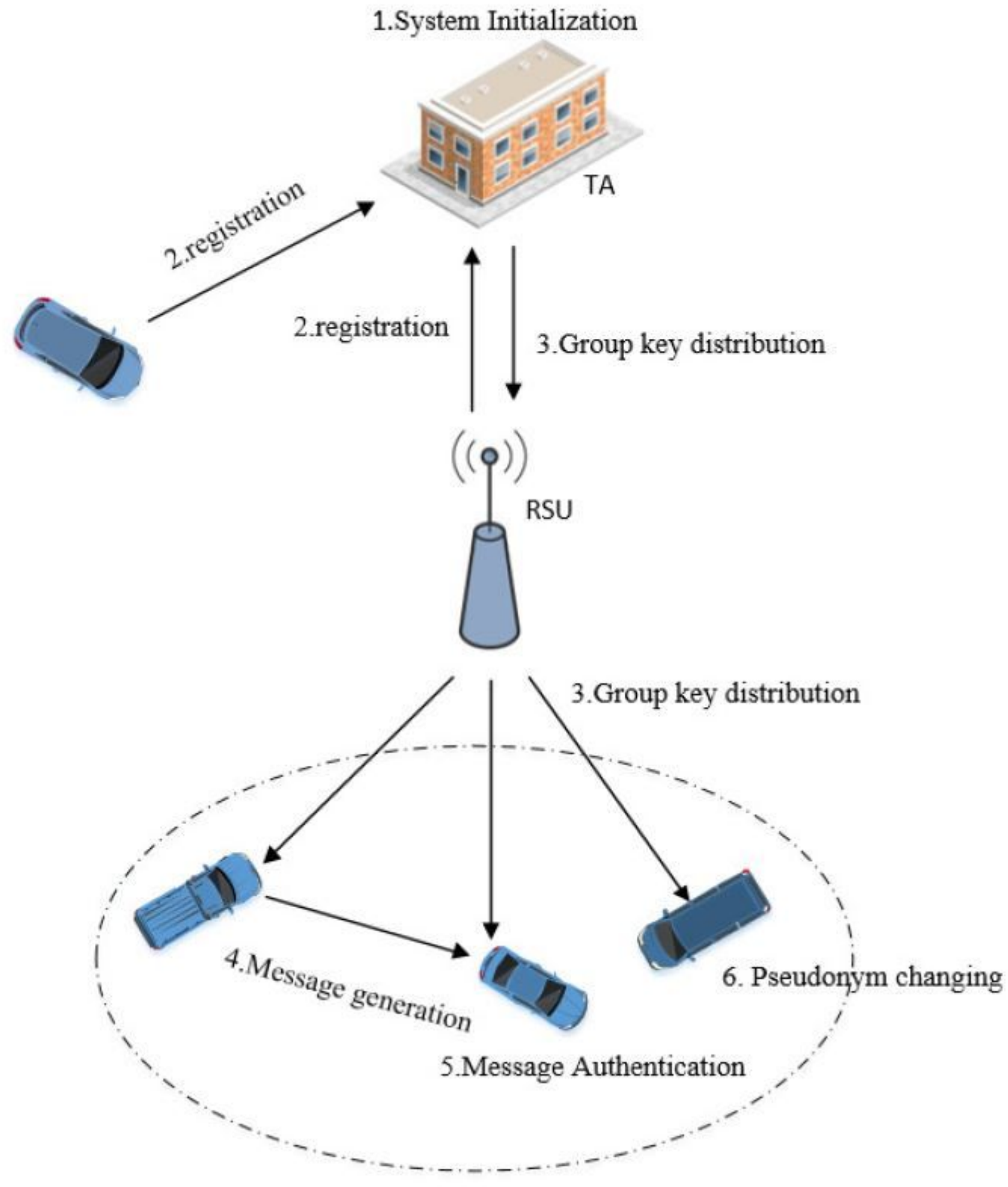

Figure 2

The system model of VANET 


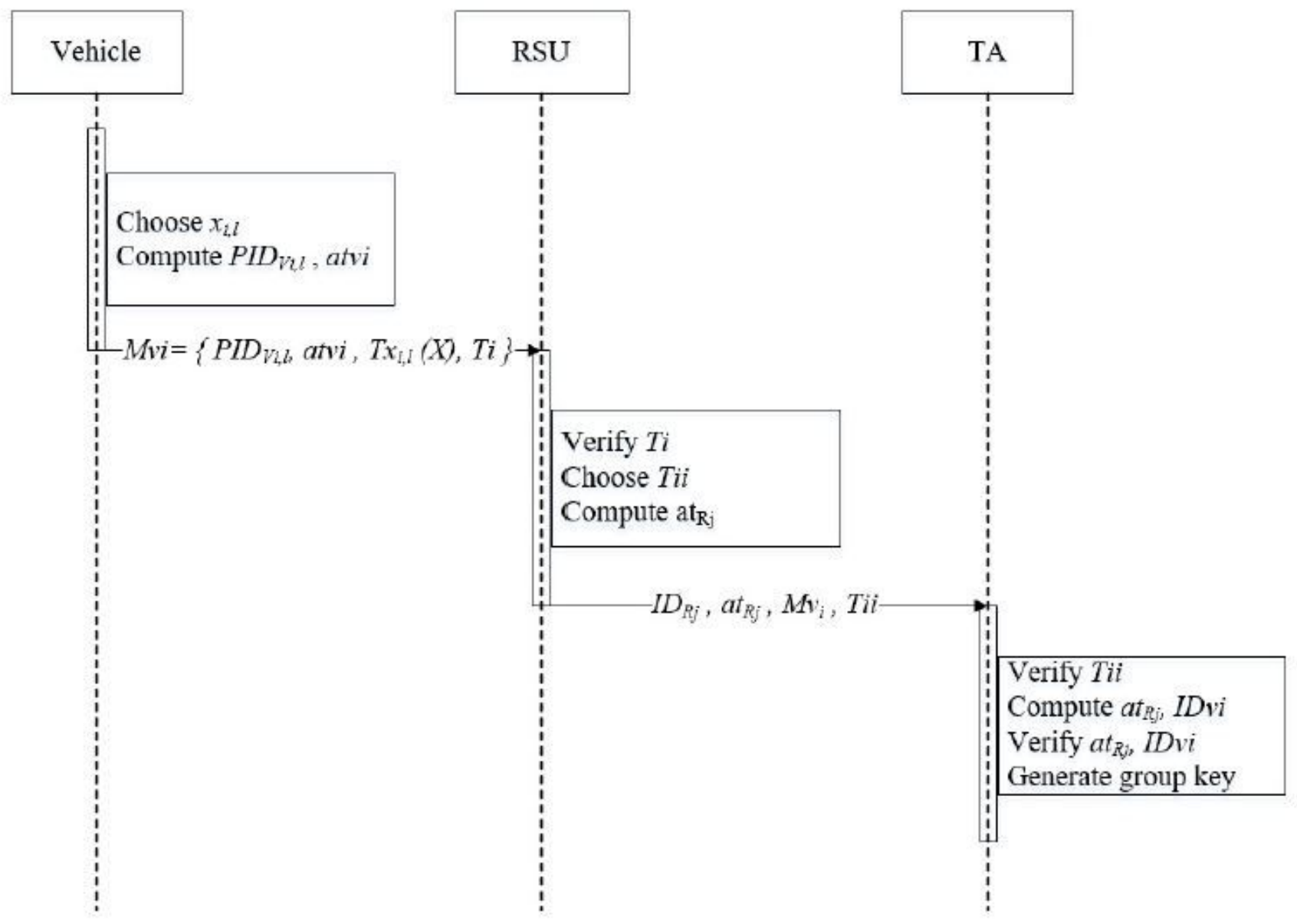

Figure 3

Vehicle authentication protocol 


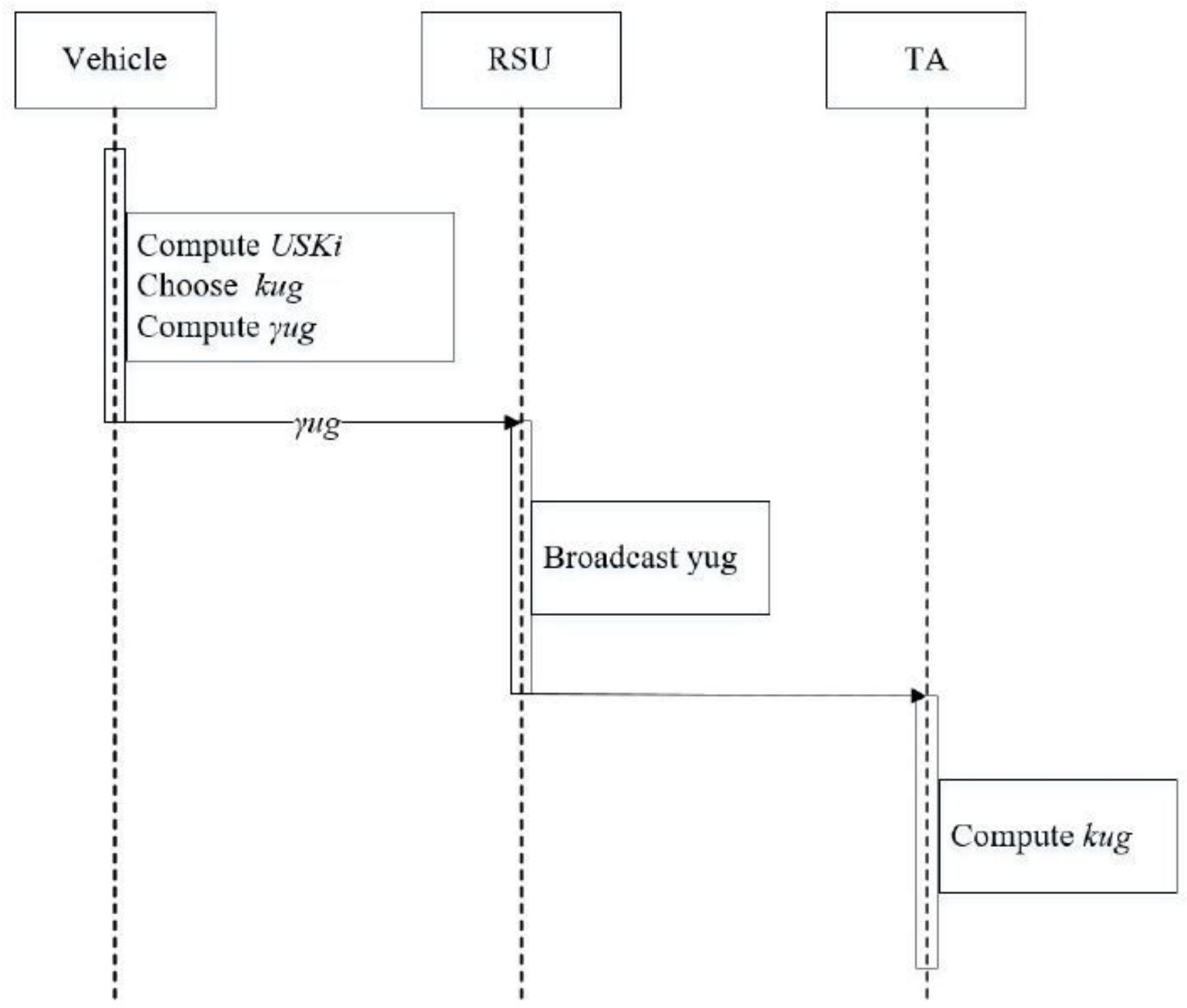

Figure 4

Group key distribution protocol 


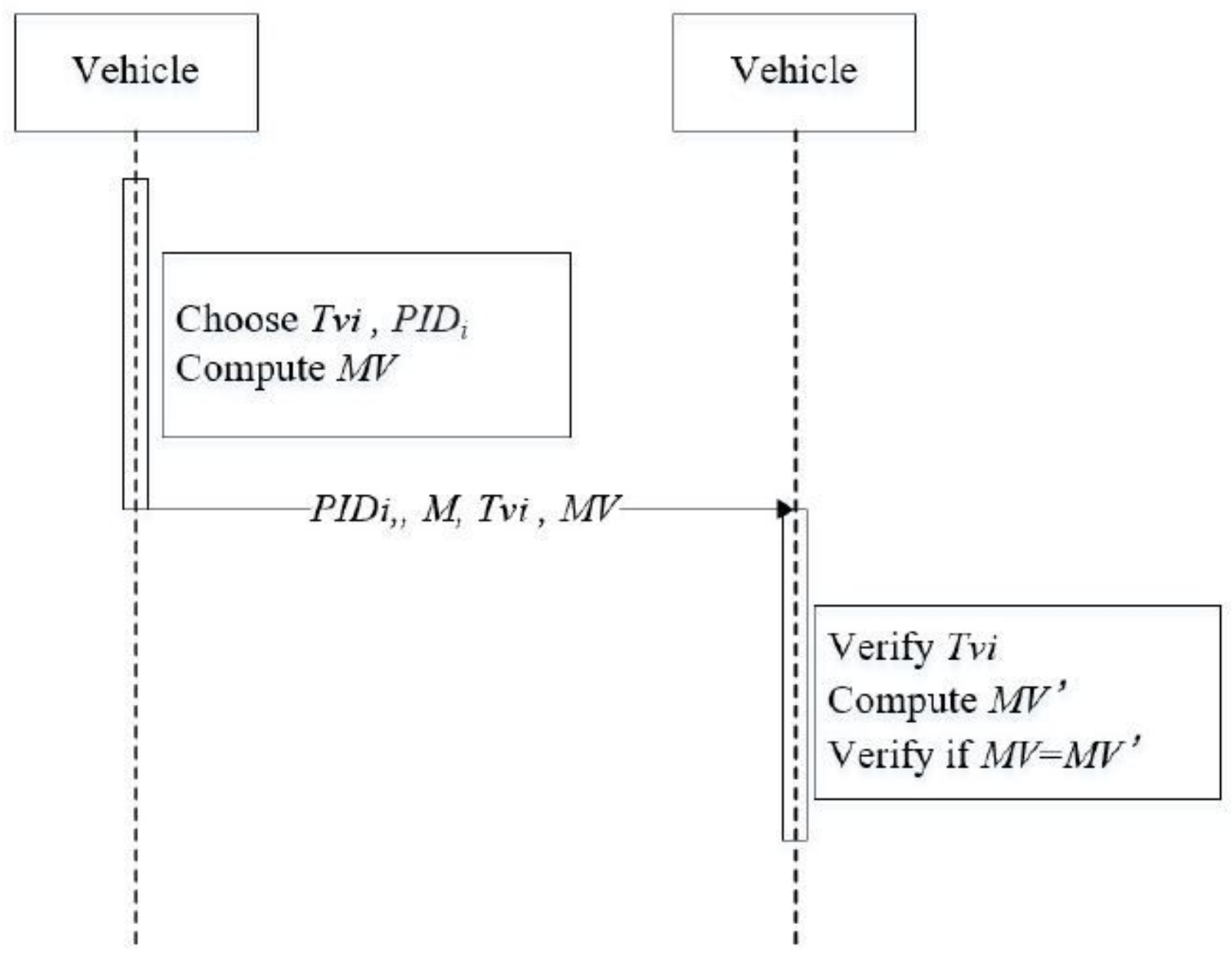

Figure 5

Message authentication protocol 